\title{
Lattice dynamics and methyl rotational excitations of 2-butyne
}

\author{
O. Kirstein and M. Prager \\ Institut für Festkörperforschung, Forschungszentrum Jülich, D-52425 Jülich, Germany \\ M. R. Johnson \\ Institut Laue-Langevin, Avenue des Martyrs, BP 156, 38042 Grenoble Cedex 9, France \\ S. F. Parker \\ ISIS facility, Rutherford-Appleton-Laboratory Chilton, Didcot OX11 OQX, United Kingdom
}

(Received 8 February 2002; accepted 23 April 2002)

\begin{abstract}
On the basis of the recently determined low temperature crystal structure the lattice dynamics of 2-butyne and a single particle methyl rotational potential are calculated using pair potential parameters given by Williams in 1974 within the model of semirigid molecules. In the regime of lattice modes the existence of four methyl librational bands with significant dispersion can explain the measured density of states. The single particle librational energy obtained for the rotational potential from the Schrödinger equation of the methyl rotor coincides well with the mean librational band energy. The calculated single particle tunneling frequency is only $17 \%$ lower than observed in experiment. Similarly well the activation energy is reproduced. Although the pair potential parameters developed for aromatic compounds need some scaling they are useful for materials with triple bond carbons. (C) 2002 American Institute of Physics. [DOI: 10.1063/1.1485729]
\end{abstract}

\section{INTRODUCTION}

The theory developed originally to describe rotational tunneling of molecular crystals is a mean field or single particle model (SPM). ${ }^{1}$ Despite the more recent detection of systems with coupling and multidimensional tunneling ${ }^{2,3}$ the prominent number of molecular crystals can be well described within the original SPM. ${ }^{4}$ A data analysis going beyond a phenomenological use of this model has to explain the rotational potential on the basis of the crystal structure and fundamental intermolecular interactions. ${ }^{5,6}$ Often intermolecular interactions are modeled as transferable intermolecular pair interactions. There is an increasing number of systems analyzed this way. Their number is mainly limited by the knowledge of a precise low temperature crystal structures of systems whose tunnel splitting is known from high resolution neutron spectroscopy. Another difficulty is due to the reliability of the atom-atom potential ansatz for the intermolecular interaction. The more complex a compound is the more difficult is it to get reliable pair potential parameters from literature. For this second reason we concentrated at the beginning our work on simple systems, mainly pure hydrocarbons as toluene ${ }^{5}$ and $p$-xylene ${ }^{7}$ which belong to a class of materials most intensively studied by the concept of pair potentials. ${ }^{8,9}$ In the present paper we want to extend the successful earlier description to an only slightly different material. 2-butyne-also known as dimethylacetylene (DMA) - is again a pure hydrocarbon but with a triple bond between the two central carbon atoms. It is the simplest compound containing at the same time methyl groups but in contrast to other hydrocarbons there are no pair potentials (PP) available from literature for this material. The question arises whether pair potentials of DMA differ from those of aliphatic or aromatic hydrocarbons.

2-butyne is one of the first systems where rotational tun- neling was observed by high resolution neutron spectroscopy. ${ }^{10,11}$ It was difficult to relate this tunnel splitting to the librational modes measured in a forthcoming experiment. ${ }^{12}$ In this paper the missing knowledge of the crystal structure did not allow a mode analysis and the description had finally to be based on an ad hoc model allowing for dispersion of the librational modes.

Thus it became interesting to study this material in a more comprehensive way after the low temperature crystal structure became known, ${ }^{13}$ see Fig. 1. The technique of choice is to combine the structural information with transferable intermolecular pair potentials. The basic questions are threefold:

(1) Is the assumption of a semirigid molecule justified?

(2) How does the phenomenological explanation of the density of states of Ref. 12 compare with a mode analysis based on a lattice dynamics calculation?

(3) Do the pair potential parameters need to be modified compared to those used for aromatic hydrocarbons ${ }^{14}$ due to triple bond between the center carbons?

\section{EXPERIMENTAL RESULTS}

\section{A. Excitations}

Inelastic neutron scattering was used to study excitations in the regime of phonons and internal excitations. Due to its wide accessible energy range up to $500 \mathrm{meV}$ and its good energy resolution $\delta E / E \sim 0.02$ in the whole spectral range the inverse time-of-flight spectrometer TFXA of the ISIS spallation source at the Rutherford-Appleton Laboratories, UK, was used. ${ }^{15}$ At this crystal analyzer instrument energy transfer and momentum transfer are correlated. The INS spectrum is available from the INS database ${ }^{16}$ at www.isis.rl.ac.uk/insdatabase. 


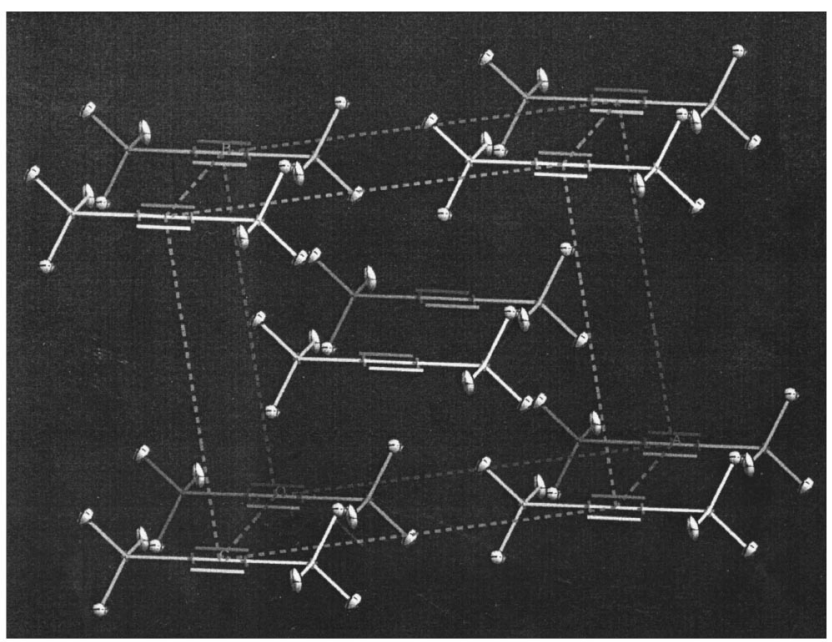

FIG. 1. Unit cell of DMA, space group $C 2 / m$ showing the triple bond between the central carbon atoms. The positions of the atoms are calculated according to Ref. 13

A flat protonated sample of volume $30 \times 50 \times 1 \mathrm{~mm}^{3}$ was cooled down to $T \leqslant 15 \mathrm{~K}$ and measured for $24 \mathrm{~h}$. This corresponds to an integrated proton dose of $2000 \mu \mathrm{Ah}$. All detectors are summed up in a single spectrum shown in Fig. 2. The spectrum decomposes into two regimes. Above about 20 $\mathrm{meV}$ internal molecular vibrations, higher harmonics and combination bands are visible. Below $20 \mathrm{meV}$ we have the regime of phonons. This part of the spectrum was measured already earlier at the direct time-of-flight spectrometer IN4 of the ILL, France. ${ }^{12}$ Small differences in peak positions are likely due to the different ways the used instruments integrate over the dispersion curves.

\section{B. Quasielastic scattering and activation energy}

The quasielastic spectra of DMA had been obtained using the FOCUS spectrometer at the Swiss neutron source SINQ. The wavelength of the incoming neutrons were chosen to be $\lambda=5.8 \AA$ with a corresponding elastic energy resolution $\delta E=50 \mu \mathrm{eV}$. The energy transfer range of interest was between -2 and $1 \mathrm{meV}$. The experimental data were

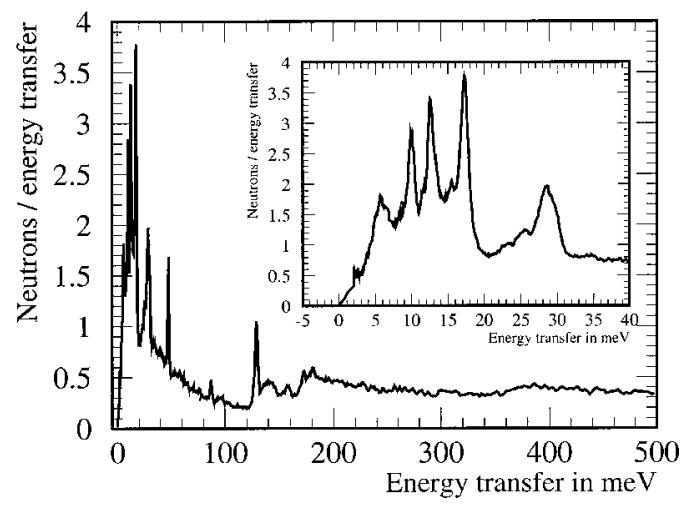

FIG. 2. Neutron spectrum of DMA up to $310 \mathrm{meV}$ measured at a sample temperature $T \leqslant 15 \mathrm{~K}$. There are no further excitations visible up to 500 $\mathrm{meV}$ likely due to recoil broadening. The regime of phonons/lattice modes below $20 \mathrm{meV}$ is shown in the insert. Four peaks at 6, 9.9, 12.6, and 17.3 $\mathrm{meV}$ are clearly visible.

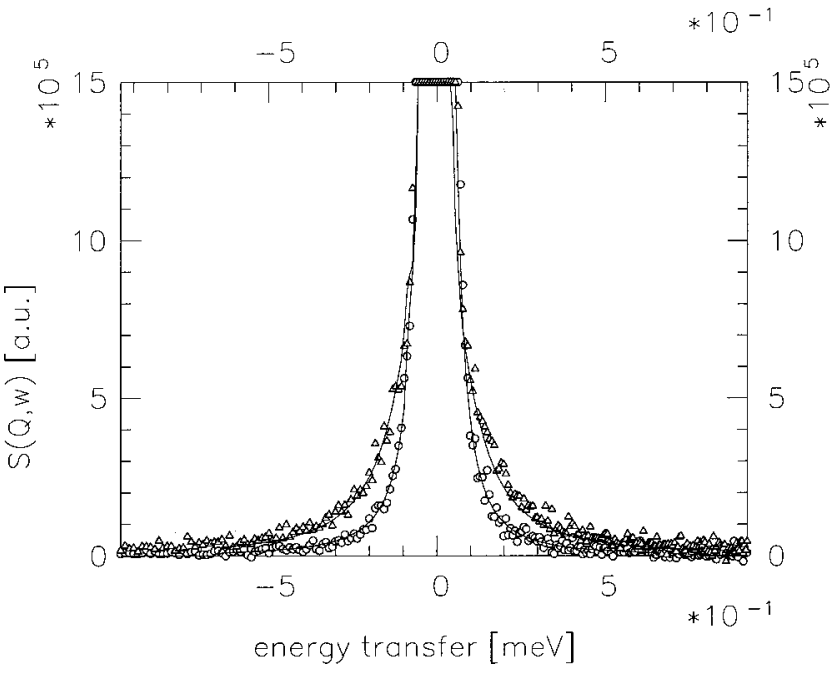

FIG. 3. Scattering function $S(Q, \omega)$ of DMA in the quasielastic regime. The temperature dependent quasielastic line broadening is shown for temperatures of $80 \mathrm{~K}(\bigcirc)$ and $120 \mathrm{~K}(\triangle)$.

transformed into $S(Q, \omega)$ using standard procedures. Figure 3 shows the broadening of the quasielastic line for two different temperatures. Since all methyl groups are crystallographical equivalent the resulting QNS spectra are described by a single Lorentzian.

The model used to analyze quasielasic spectra is a simple hopping model. Since the potential forces the molecule into the equilibrium position the only possibility for a hydrogen to move is to jump over the barrier into an equivalent position. The scattering function $S(Q, \omega)$ for a powder sample with protons jumping on a triangle with a jump frequency $\nu$ is given by

$$
S(Q, \omega)=A_{0}(Q) \delta(\omega)+A_{1}(Q) \frac{1}{\pi} \frac{\frac{3}{2} \nu}{\left(\frac{3}{2} \nu\right)^{2}+\omega}
$$

with the prefactors $A_{0}(Q)=\frac{1}{3}\left(1+2 j_{0}(Q d)\right)$ and $A_{1}(Q)$ $=\frac{2}{3}\left(1-j_{0}(Q d)\right)$, where $Q$ is the value of the scattering vector and $d$ the jump length, i.e., the proton-proton distance of $1.78 \AA$ inside the $\mathrm{CH}_{3}$ group. The width of the Lorentzian in Eq. (1) is $\Gamma=(3 / 2) \nu$. Since jumps are thermally activated processes the jump frequency and thus the linewidth of the Lorentzian depends on the temperature and follows an Arrhenius behavior,

$$
\begin{aligned}
\Gamma(T) & =\Gamma_{0} \cdot \exp \left(-\frac{E_{a}}{k T}\right) \\
& =\Gamma_{0} \cdot \exp \left(-0.0116 \cdot E_{a}[\mathrm{meV}] \cdot \Theta\left[K^{-1}\right]\right)
\end{aligned}
$$

with $\Theta=10^{3} / T$.

The hopping model allows to derive the activation energy $E_{a}$ which yields the energy difference between the ground state and the maximum of the methyl rotational potential.

In order to get the activation energy eight quasielastic spectra were collected in the temperature range of $70 \mathrm{~K} \leqslant T$ $\leqslant 150 \mathrm{~K}$ (corresponding to $6.7 \mathrm{~K}^{-1} \leqslant \Theta \leqslant 14.3 \mathrm{~K}^{-1}$ ) and analyzed with respect to the temperature dependent FWHM of the Lorentzians. A least squares fit to the data yields $E_{a}$ 


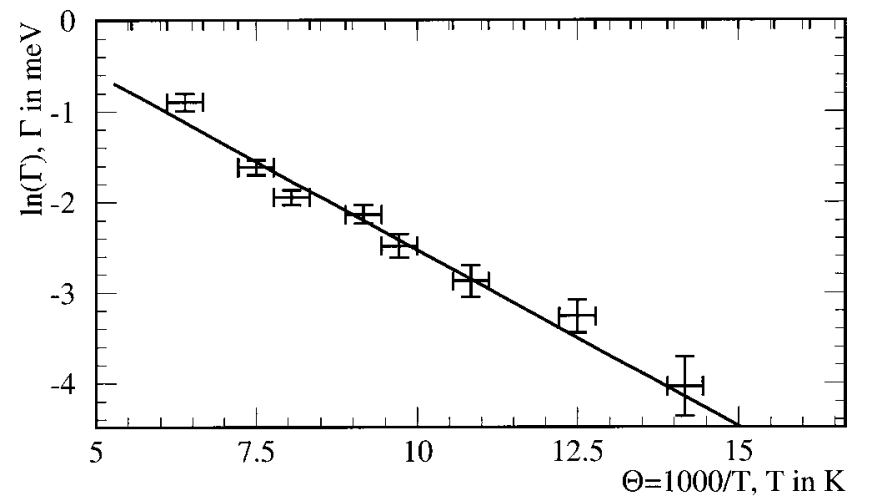

FIG. 4. Arrhenius plot $\Gamma(\Theta)=\Gamma_{0} \cdot \exp \left(-0.0116 \cdot E_{a} \cdot \Theta\right)$ of the temperature dependent FWHM $T$ of the incoherent quasielastic line broadening, see also Fig. 3.

$=33.6 \mathrm{meV}(3.2 \mathrm{~kJ} / \mathrm{mol})$, Fig. 4 . The prefactor $\Gamma_{0} \approx 4 \mathrm{meV}$ is consistent with the attempt frequency found otherwise for methyl groups.

\section{CALCULATIONS}

Molecular crystals are most often described by the model of rigid or semirigid molecules: To reduce the number of degrees of freedom (DOF) all or most internal DOF are frozen. Indeed very often the hard internal vibrations do not overlap with the external modes. For molecules with attached rotating radicals like methyl groups, however, only semirigidity can be valid. The soft internal modes are now included into the external DOF. Ab initio calculations give a quantitative measure of the internal DOF which have to be included by the lattice dynamical calculation.

\section{A. Ab initio calculations for the isolated molecule}

In the gas phase, there is free rotation of the methyl groups, thus the $D_{3 h}$ (methyl groups eclipsed) and $D_{3 d}$ (methyl groups staggered) structures are equivalent. In the low temperature $C 2 / m$ phase ${ }^{13}$ the center of the molecule lies on a point of inversion, however, the molecule is slightly deformed, such that one of the $\mathrm{C}-\mathrm{H}$ bonds is shorter than the other two and the symmetry is reduced to $C_{2 h}$.

The vibrational modes of molecules containing up to a few tens of first row atoms can be calculated on a PC using $a b$ initio methods. In the present case, density functional theory (DFT) as implemented in the GAUSSIAN 98 (Ref. 17) package was used with the B3LYP functional and 6-31G, 6-311+ $\mathrm{G}$, and $6-311+\mathrm{G}(d, p)$ basis sets. All the methods yielded similar results, with the largest basis set providing the best results.

The $D_{3 h}, D_{3 d}$ structures and a $C_{2 h}$ structure that used the crystallographic geometry as the initial input were examined. All gave stable structures, although in each case, the antiphase methyl torsion was close to zero. The $C_{2 h}$ structure converged to a geometry that was almost $D_{3 d}$, this is also shown by the very small $(<0.1 \mathrm{meV})$ splitting of the degenerate modes. This result strongly suggests that the solid state structure is crystallographically imposed.

The observed intensity of an INS band is given by ${ }^{18}$

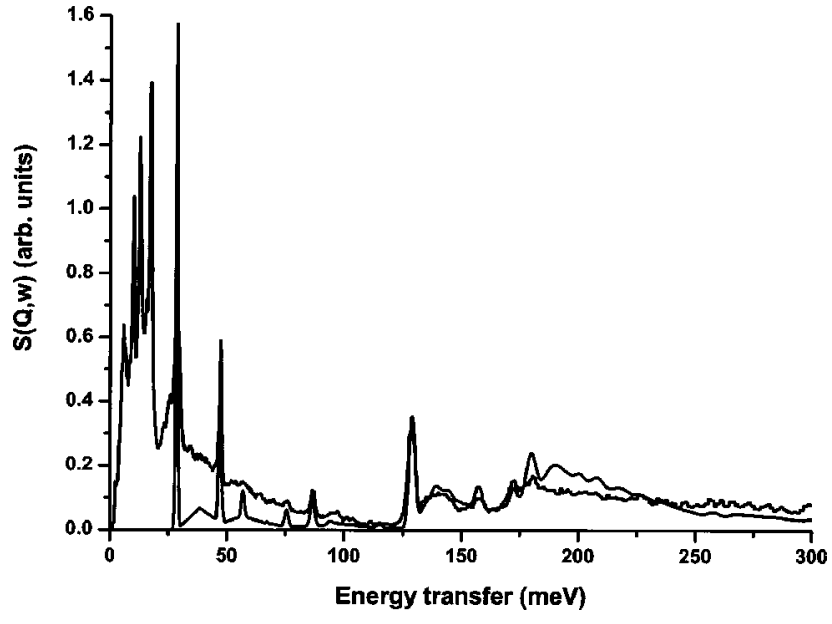

FIG. 5. Comparison between the measured TFXA and the calculated a-CLIMAX spectra, based on the calculated atomic displacements. See text for details.

$$
S\left(Q, n \omega_{i}\right) \propto \frac{\left(Q \cdot U_{i}\right)^{2 n}}{n !} \exp \left(-\left(Q \cdot U_{\mathrm{Tot}}\right)^{2}\right) \cdot \sigma,
$$

where $\omega_{i}$ is the $i$ th mode at frequency $\omega, n=1$ for a fundamental, 2 for a first overtone or binary combination, 3 for a second overtone or ternary combination etc., $Q$ is the momentum transfer defined earlier, $U_{i}$ is the root mean square displacement of the atoms in the mode, $U_{\text {Tot }}$ is the total root mean square displacement of all the atoms in all the modes, both internal and external, $\sigma$ is the inelastic scattering cross section of the atom. The total scattering cross sections of hydrogen and carbon are 82.0 and 5.6 barns ( 1 barn $=1$ $\times 10^{-28} \mathrm{~m}^{2}$ ), respectively ${ }^{19}$ and since the intensity is dependent on the amplitude of vibration, which is larger for light atoms, the spectra will be dominated by motions that involve displacement of hydrogen. The exponential term in Eq. (2) is a Debye-Waller factor. Equation (2) is based on an isolated molecule approximation, thus dispersion is not included. The narrow linewidths of the modes above $25 \mathrm{meV}$ demonstrate that this is a reasonable assumption in the present case.

The output of GAUSSIAN 98 includes the atomic displacements for each mode, which can be used to visualize the mode and also to generate the INS spectrum. The program a-CLIMAX (Ref. 20) was used for this purpose. After minor adjustment of the frequencies to match the observed values and inclusion of events involving up to three quanta the result shown in Fig. 5 is obtained. The agreement between observed and calculated is excellent confirming the assignments given in Table I.

The width of the skeletal bending mode at $39.7 \mathrm{meV}$ is significantly larger than the resolution function which results in an apparent mismatch for this band, although the integrated intensity is approximately correct. This is probably due to factor group splitting since there are two molecules in the unit cell. This frequency is significantly above the phonon energies so justifies the treatment of the molecule as a rigid body in the lattice dynamical calculations described in the following section. 
TABLE I. Vibrational modes of DMA as calculated $a b$ initio using the GAUSSIAN 98 program are compared to the excitation energies measured by neutron spectroscopy. Modes are assigned to each excitation energy.

\begin{tabular}{cclc}
\hline $\begin{array}{c}\text { Gaussian } \\
\text { energies } \\
(\mathrm{MeV})\end{array}$ & Multiplicity & \multicolumn{1}{c}{ Mode character } & $\begin{array}{c}\text { Observed (TFXA) } \\
\text { energies (meV) }\end{array}$ \\
\hline 0 & 1 & methyl torsion & $6,9.9,12.6,17.3$ \\
26.4 & 2 & backbone bend & 28.5 \\
47.1 & 2 & backbone twist & 47.4 \\
87.5 & 1 & backbone sym. stretch & 87 \\
132 & 4 & methyl rock & 129 \\
141 & 1 & backbone asym. stretch & 172 \\
176 & 2 & methyl sym. breath. & 181 \\
186 & 4 & methyl deformation & \\
290 & 1 & backbone stretch & \\
$378-386$ & 6 & proton stretch & \\
\hline \hline
\end{tabular}

The strong mode at $129 \mathrm{meV}$ is the methyl rocking mode and both the INS spectrum and the $a b$ initio results show that the four (two doubly degenerate modes) are coincident. The low temperature Raman spectrum ${ }^{21}$ shows that the asymmetric methyl bending modes give rise to a broad, $\sim 5 \mathrm{meV}$, band at $180 \mathrm{meV}$. The ab initio results suggests that these bands are very close in energy which is why the feature is apparently overestimated. Inspection shows that there is missing intensity in the calculated spectrum at $175 \mathrm{meV}$, suggesting that one of the modes is calculated too high in energy. Shifting one of the modes to lower energy would also reduce the overestimation of the phonon wings that are responsible for the intensity at $\sim 185 \mathrm{meV}$.

The higher energy modes are poorly resolved from the phonon wings. This is due to the large Debye-Waller factor that results from a relatively light molecule and the large displacements in the coupled torsional/librational modes. Since the $\mathrm{C} \equiv \mathrm{C}$ stretch has little proton motion, it is intrinsically weak in the INS spectrum and is not observed. At large energy transfers, the spectral detail is obliterated by molecular recoil. There is nearly no internal torsional restoring force. The next lowest eigenenergies around $26 \mathrm{meV}$ belongs to a bend of the molecule backbone in two different but equivalent planes. Thus the lowest internal vibrational mode energy is (see below) above the largest phonon energies. Thus we describe the molecule in the following lattice dynamical calculations as completely rigid beside of the internal torsion.

\section{B. Lattice dynamics of the crystal}

Phonon dispersion curves are calculated by the program DYNCAL which was successfully used for similar applications earlier. ${ }^{5,7,22}$ With DYNCAL phonon energies and eigenvectors are obtained by diagonalizing the dynamical matrix. DMA contains two molecules in the unit cell with one soft internal degree of freedom each, the out-of-phase methyl librations. For otherwise rigid molecules this yields 14 phonon branches. The force constants are derived in harmonic approximation as second derivatives of atom-atom pair potentials. The intermolecular interactions were modeled in the following by a Born-Meyer potential:
TABLE II. Williams'74 and scaling parameters used to calculate the lattice dynamics of DMA.

\begin{tabular}{cccccl}
\hline \hline Pair & $A_{i j}(\mathrm{kcal} / \mathrm{mol})^{\mathrm{a}}$ & $B_{i j}\left(10^{3} \mathrm{kcal} / \mathrm{mol}\right)$ & $\alpha_{i j}\left({ }^{-1}\right)$ & $k_{i j}$ & \multicolumn{1}{c}{$f$} \\
\hline $\mathrm{H}-\mathrm{H}$ & 40.1 & 2.876 & 3.74 & 1.012 & for all \\
$\mathrm{H}-\mathrm{C}$ & 134.2 & 14.310 & 3.67 & 0.700 & types \\
$\mathrm{C}-\mathrm{C}$ & 449.0 & 71.430 & 3.60 & 0.484 & 1.016 \\
\hline
\end{tabular}

${ }^{\text {a Reference } 6 .}$

$$
V_{i j}(r)=k_{i j} \cdot\left(\frac{A_{i j}}{\left(f \cdot r_{i j}\right)^{6}}+B_{i j} \cdot \exp \left(\alpha_{i j} \cdot\left(f \cdot r_{i j}\right)\right)\right),
$$

where $i j$ stands for the respective atom-pair $(\mathrm{H}-\mathrm{H}, \mathrm{H}-\mathrm{C}$, and $\mathrm{C}-\mathrm{C}) . A, B$, and $\alpha$ are pair potential parameters given by Williams $^{14}$ (referred as Williams'74) and $k$ and $f$ are scaling parameters. These parameters allow one to adjust the potential which may be necessary since Williams derived the potential parameters for the class of aromatic molecules not containing carbon-carbon triple bonds. It is obvious that $V_{i j}(r)$ represents the classical Born-Meyer potential for $k_{i j}$ $=f=1$. The values used for the lattice dynamical calculations are given in Table II. They follow the condition of systematic transferability $k_{i j}=\sqrt{k_{i i} \cdot k_{j j}}$. The electrostatic interaction had been taken into account by an additional potential term

$$
V_{i j}^{\mathrm{el}}(r)=\frac{n_{i} \cdot n_{j} \cdot q_{0}^{2}}{r_{i j}}
$$

Here $n_{i}$ and $n_{j}$ are the charges in units of the elementary charge $q_{0}$. Values for the partial charges are obtained from the $a b$ initio calculations for the isolated molecule, see above and Fig. 6.

For technical reasons the lattice dynamical program requires the pair potentials as a power series. ${ }^{23}$ The use of four terms of the series allowed a very good description for interatomic distances above $2.2(2.0) \AA$ for $\mathrm{C}-\mathrm{C}(\mathrm{H}-\mathrm{H}, \mathrm{C}-\mathrm{H})$ interactions.

Beside the pair potentials the lattice dynamical program requires the crystal structure to calculate the interatomic distances. The recently measured low temperature crystal structure $^{13}$ is used.

The molecule is modelled as a linear semirigid one used with negligible internal torsional restoring force. While the $a b$ initio calculation supports this description the observed deformation of the molecule - the carbon-backbone is bent in the crystal by $\pm 1.5^{\circ}$ - means that there is a coupling between external and internal modes. An analysis shows, however, that the overall behavior in the regime of lattice modes is unchanged if the molecular bend is frozen or not.

The program allows one to apply an internal restoring force to the methyl libration. This manipulation influences almost only the two librational modes with antiphase rotation of the two methyl groups of the same molecule. The in-phase modes are not shifted in energy under this action. A weak internal methyl-methyl coupling of $0.001 \mathrm{mdyn} / \AA$ was chosen. Under the given assumptions the librational modes show energies of 4.2, 11.4, 20.0, $22.8 \mathrm{meV}$ at the $\Gamma$-point. 


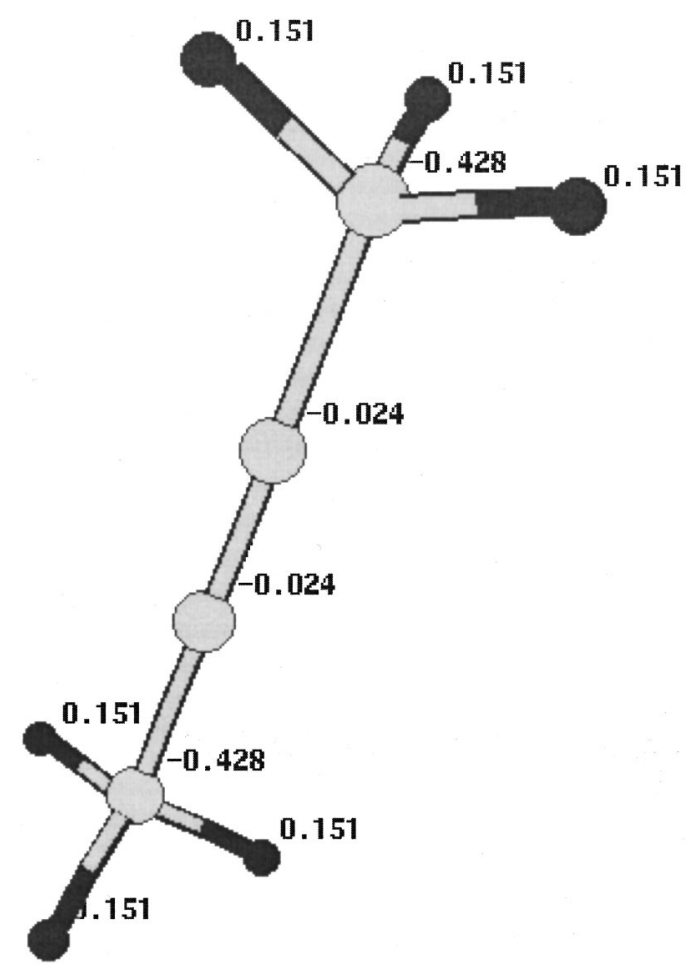

FIG. 6. Calculated charges in units of the elementary charge $q_{0}$ of the $\mathrm{H}$ and $\mathrm{C}$ atoms of DMA. The charges are derived $a b$ initio, see text.

There is huge dispersion along the $[0,1,0]$ direction, little along $[1,0,0]$. In both these directions the dispersion curves looks as if the unit cell would be a factor 2, too large. In $[0,0,1]$ direction the phonon branches flatten at the zone border yielding additional van Hove anomalies. Figure 7 shows the dispersion curve along the [001]-direction. The four methyl groups in the unit cell require four modes with a librational character corresponding to an in-phase rotation of methyl groups inside a molecule and an in-phase rotation of the two molecules in the unit cell (in-in) and correspondingly in-out, out-in, and out-out. Figure 8 shows a view of the eigenvectors of the out-out-mode. Numerical results are summarized in Table III. They yield already a qualitative explanation of the multiline phonon spectrum of Fig. 2.

The lattice dynamical program contains all ingredients to calculate the neutron scattering function $S(Q, \omega)$ in the meV regime — or equivalently the DOS—as a weighted average of all excitations,

$S(Q, \omega) \sim Q^{2} \sum_{i} \sigma_{i} \sum_{l} \sum_{j}\left\langle u_{i j l}^{2}\right\rangle \cdot D W(u) \cdot \delta\left(\omega-\omega_{j}\left(q_{l}\right)\right)$.

The summation runs again over all displacements $u$ of $2 \cdot 10$ atoms $i$, the 14 modes $j$ and a homogeneous grid of momentum transfers $q_{l}$ of the Brillouin zone and includes the Debye-Waller factor DW $(u)$. The only difference to the spectrum of the internal modes, Eq. (2), consists of an additional summation $l$ over the Brillouin zone required by phonon dispersion. Methyl librations are strongly anharmonic and show the largest mean square displacements. Further they invoke the proton with its dominant scattering cross section $\sigma_{i}$ and causing the strongest peaks on the DOS. Fig-

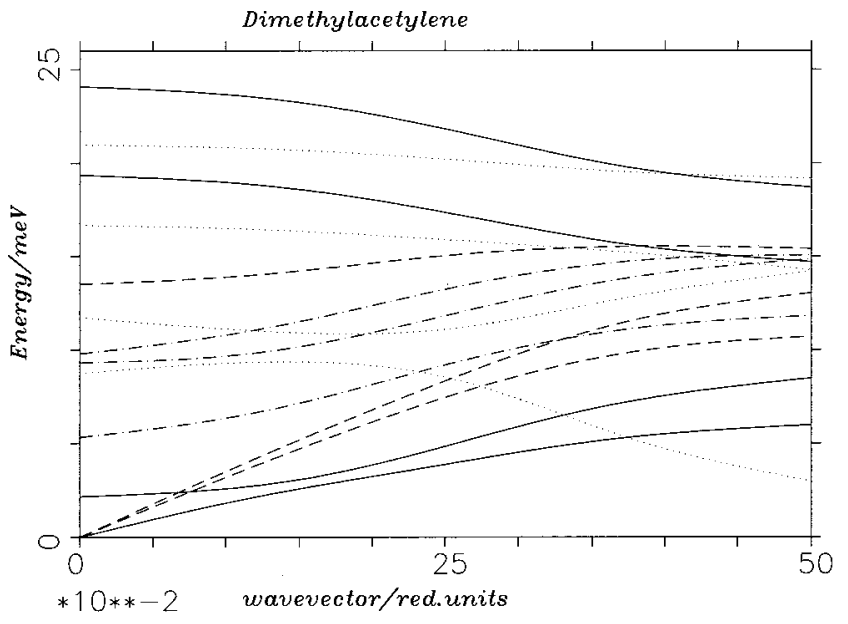

FIG. 7. Dispersion curve of DMA along the [001]-direction. Pair potential parameters are taken from Williams' 74 with the modifications according to Eq. (3) and Table II.

ure 9 shows the result of such a calculation. There is good agreement between the calculated (shaded) and measured (open dots) DOS although there are differences in the intensities of the peaks especially at $\sim 6 \mathrm{meV}$ and $\sim 13 \mathrm{meV}$.

In Tilli's work ${ }^{12}$ it was the lack of knowledge of the low temperature crystal structure which allowed only a phenomenological access to the problem. The authors introduced a rotor-rotor coupling between neighboring methyl groups and therewith dispersion to explain two peaks of the DOS. Required parameters were chosen arbitrarily to fit the maxima at 12.9 and $17.7 \mathrm{meV}$ of the measured DOS. This led to an average librational energy of $E_{01}$ and a rotational single particle potential which is consistent with the observed tunnelling splitting, see Table IV.

\section{Methyl rotational potentials}

Besides the DOS, the rotational tunneling of the methyl group must be described by the chosen transferable pair potential, too. For this purpose a mean field rotational potential has to be calculated using the same pair interactions as for the lattice dynamics. A methyl group is rotated about the bond axis while all other atoms are fixed at their equilibrium positions. Adding up, for each $\mathrm{CH}_{3}$ orientation, the pair interactions of the three methyl protons with all the other lattice atoms yields the potential energy of this configuration.

It contains an angle dependent part which can be identified as the mean field methyl rotational potential $V(\varphi)$. For further analysis the angle dependent potential energy of the crystal is described by Fourier expansion,

$$
V(\varphi)=V_{0}+\sum_{n=1}^{2} \frac{V_{3 n}}{2}\left(1-\cos \left(3 n \varphi+\varphi_{n}\right)\right) .
$$

The rotational potential has exactly threefold symmetry since the methyl group relaxes into identical equilibrium orientations after each $2 \pi / 3$ rotation. If one would rotate the $\mathrm{CH}_{3}$ group as determined crystallographically with all the positional error bars as a rigid unit the proton sites would coincide only after $2 \pi$ rotation. Thus when calculating $V(\varphi)$ we force the methyl protons to move on the common circle of 
Mode $(1,1): 9$ Emev=11.3603 $q=0.0000 .0000 .000$

Dimethylacetylene

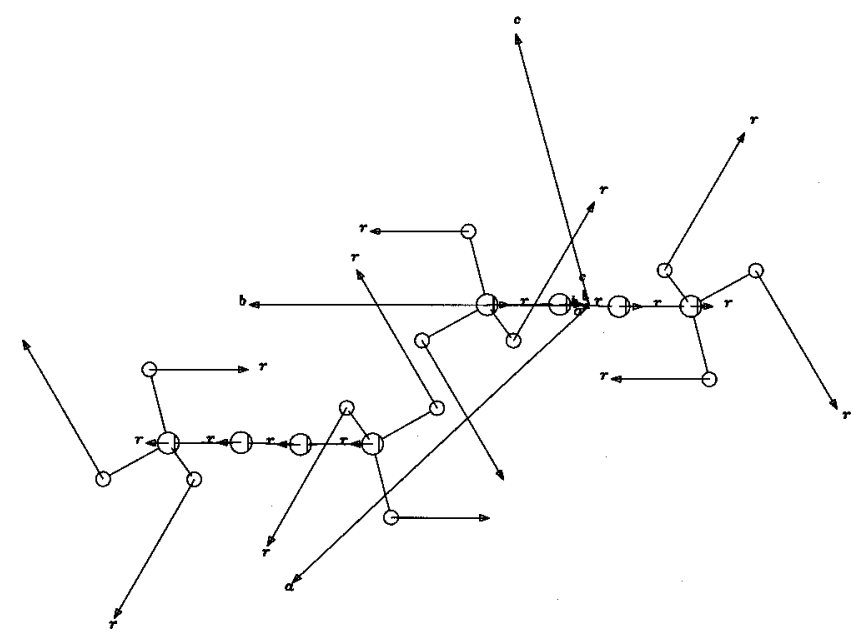

FIG. 8. Out-out eigenmode of energy $11.4 \mathrm{meV}$ at the $\Gamma$ point.

circumference between equilibrium positions of the two neighboring methyl protons. This procedure makes the protons coincide exactly after $2 \pi / 3$. The resulting potential is shown in Fig. 10 for $0^{\circ} \leqslant \varphi \leqslant 120^{\circ}$. The main contribution to $V(\varphi)$ stems from atoms within a radius of about $6 \AA$. Two features are visible in Fig. 10. At first, the potential minimum coincides well with the crystallographic orientation of the methyl group as required for a stable system. Secondly, the potential is exactly symmetric to the maximum. This is due to the presence of the $a-c$ mirror plane intersecting the methyl group in the $C 2 / m$ space group. The potential has a minimum flatter than harmonic, a somewhat narrower large maximum indicating a contribution with sixfold symmetry and coinciding maxima of the two Fourier terms.

The potential determines the rotational excitations of the methyl group-tunneling, librations etc.--through the single particle Schrödinger equation of the one-dimensional threefold rotor,

$$
H=-B \frac{\partial^{2}}{\partial \varphi^{2}}+V(\varphi) .
$$

$B=0.655 \mathrm{meV}$ is the rotational constant of the rotating methyl group about the rotation axis. The broadened minimum

TABLE III. Calculated libron energies according to Eq. (3) and Table II. The mode characters are described by the phase relation within one molecule and between the two molecules in the unit cell. For example "in-in" means, that all methyl groups rotate in phase.

\begin{tabular}{ccl}
\hline \hline$q$-point & Energy $(\mathrm{meV})$ & Character \\
\hline$(0,0,0)$ & 4.2 & in-in \\
& 11.4 & out-out \\
& 20.0 & out-out \\
& 22.8 & in-in \\
$(0,0,0.5)$ & 4.9 & in-in \\
& 8.7 & out-in \\
& 18.0 & in-in \\
& 18.7 & out-out \\
\hline \hline
\end{tabular}

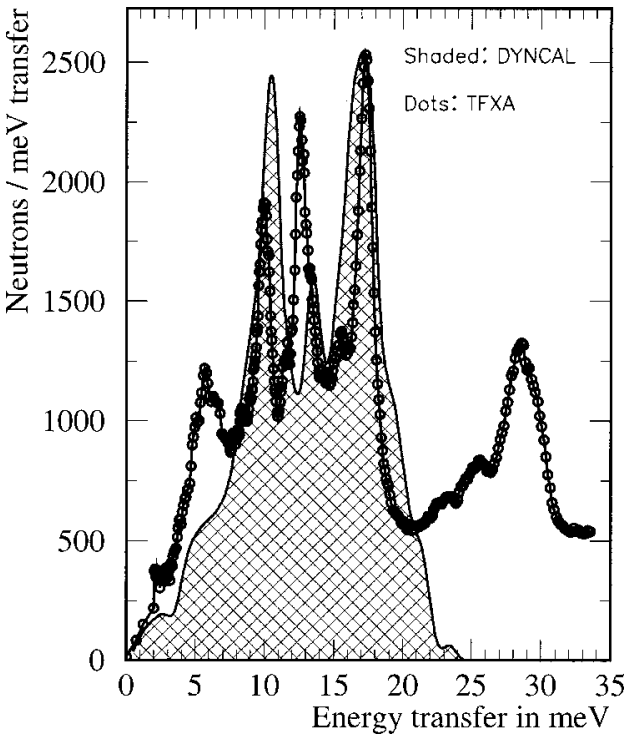

FIG. 9. Scattering function of DMA in the meV regime as calculated from the crystal structure using Williams'74 pair interaction parameters.

of the potential leads to lowering of the librational modes compared to a pure $\cos (3 \varphi)$ potential. The calculated librational energy of $E_{01}=14.16 \mathrm{meV}$ has no direct counterpart in the observed DOS but the calculated value of $E_{01}$ can be interpreted as an average of the dispersive librational bands. The value is slightly lower than the experimental value of 15 $\mathrm{meV}$ derived by Kollmar and Alefeld ${ }^{11}$ but still in good agreement. The tunnel frequency of $\hbar \omega_{t}=1.45 \mu \mathrm{eV}$ coincides within $17 \%$ with the observed tunnel splitting of 1.74 $\mu \mathrm{eV} .^{10}$ The derived rotational potential is further confirmed by the quasielastic scattering experiment. The measured activation energy $E_{a}$ differs from the calculated one by only $6 \%$. Table IV shows the librational and tunnel energies together with the extracted potential parameters. The parameters of the fitted rotational potential are shown in the lowest line of the table.

An artificial increase of the size of the unit cell and a restriction to one molecule per unit cell allows one to simulate the case of the isolated molecule with the lattice dynamical program. In agreement with the GAUSSIAN 98 result the rotational potential is completely removed under these con-

TABLE IV. Comparison between observed energies and calculated ones (tunneling, libration, activation, and potential). Experimental values are boldfaced, calculated ones are italic.

\begin{tabular}{|c|c|c|c|c|c|}
\hline $\begin{array}{l}\hbar \omega_{t} \\
(\mu \mathrm{eV})\end{array}$ & $\begin{array}{c}E_{01} \\
(\mathrm{meV})\end{array}$ & $\begin{array}{c}V_{3} \\
(\mathrm{meV})\end{array}$ & $\begin{array}{c}V_{6} \\
(\mathrm{meV})\end{array}$ & $\begin{array}{c}E_{a} \\
(\mathrm{meV})\end{array}$ & Origin \\
\hline \multirow[t]{2}{*}{1.74} & & 45.0 & & & Alefeld and Kollmar ${ }^{\mathrm{a}}$ \\
\hline & 15 & 45.0 & & & Kollmar and Alefeld ${ }^{b}$ \\
\hline \multirow[t]{3}{*}{1.74} & 15.2 & 43.3 & 3.5 & & Tilli and Alefeld ${ }^{c}$ \\
\hline & $6-17.3$ & & & & Experiment, TFXA \\
\hline & & & & 33.6 & Experiment, FOCUS \\
\hline 1.45 & 14.16 & 50.5 & -3.9 & 35.2 & This work, calculation \\
\hline
\end{tabular}

${ }^{\mathrm{a}}$ Reference 10 .

${ }^{\mathrm{b}}$ Reference 11.

${ }^{\mathrm{c}}$ Reference 12 . 


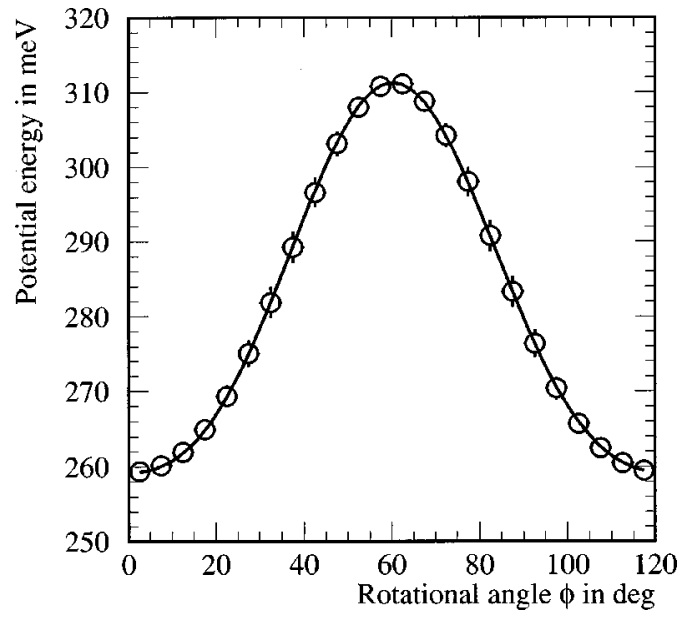

FIG. 10. Rotational potential of the methyl group based on Williams'74 pair potentials. The equilibrium orientation of the rotated $\mathrm{CH}_{3}$ group in the crystal is well reproduced by the pair potentials used. $\phi=0^{\circ}$ corresponds to the equilibrium position.

ditions and the methyl group of the isolated molecule in the lattice dynamical model represents a perfect free rotor.

The case of an isolated dimer can be studied in a similar way. Due to reduced symmetry there are two types of methyl groups in this case. The interesting one is the one with the short distance to the triple bond carbon atoms (the other is again close to a free rotor). Here the potential has reached already $40 \%$ of its final strength in the crystal but its minimum is out of phase by $\sim 60^{\circ}$ from the crystallographic equilibrium orientation. Only the phase-shifted superposition of interactions with all neighbors leads to consistency. The relatively weak rotational potential results from such compensation effects. An analysis of interatomic distances or the view along the molecular symmetry axis shows that the triple bound carbon belongs to the atoms nearest to the $\mathrm{CH}_{3}$ group. Thus they give an important contribution to the rotational potential and tunneling probes especially among all others the hydrogen-carbon(3) atom-atom potential.

\section{CONCLUSION}

For a long time it was impossible to merge rotational tunneling and librational peaks of the density of states within the single particle model. Based on the measured crystallographical structure the idea that rotor-rotor-coupling causes this discrepancy suggested by Tilli could be confirmed and elaborated. This shows that it may be misleading to identify a peak in the density of states with a single particle methyl librational excitation as it is done often. The analysis of the quasielastic spectra based on classical jump rotation yields the correct barrier height (activation energy $E_{a}$ ). Thus it may be better to base an analysis on tunneling and quasielastic scattering instead of tunneling and misinterpretable raw data of the density of states.

The suspicion that DMA requires different pair potentials due to its triple bound carbon atoms was confirmed. Only a modified set of the Williams' 74 pair potential parameters used reproduce successfully not only the density of states but also the rotational tunneling, mean librational energy and activation energy of the respective methyl group. Since the triple bound carbons belong to the nearest neighbors of the methyl protons this interaction is really tested with DMA. From this point the Williams parameters seem to be a good starting point to describe hydrocarbon systems which are not single or $\pi$ bonded.

\section{ACKNOWLEDGMENTS}

The authors thank J. Tomkinson, RAL, for a first analysis of the TFXA data and M. Monkenbusch, FZJ, and W. Griessl, University of Bayreuth, for the supply of and help with the lattice dynamics program.

${ }^{1}$ W. Press, Single Particle Rotations in Molecular Crystals, Springer Tracts in Modern Physics (Springer, Berlin, 1981), Vol. 81.

${ }^{2}$ P. Schiebel, A. Hoser, W. Prandl, G. Heger, W. Paulus, and J. Schweiß, J. Phys.: Condens. Matter 6, 10989 (1994).

${ }^{3}$ V. A. Benderskii, E. V. Vetoshkin, S. Yu. Grebeshchikow, L. von Laue, and H. P. Trommsdorff, Chem. Phys. 219, 143 (1997).

${ }^{4}$ M. Prager and A. Heidemann, Chem. Rev. 97, 2933 (1997).

${ }^{5}$ M. Prager, M. Monkenbusch, R. M. Ibberson, W. I. F. David, and D. Cavagnat, J. Chem. Phys. 98, 5653 (1993).

${ }^{6}$ M. R. Johnson, M. Neumann, B. Nicolai, P. Smith, and G. J. Kearley, Chem. Phys. 215, 343 (1997).

${ }^{7}$ M. Prager, W. I. F. David, and R. M. Ibberson, J. Chem. Phys. 95, 2473 (1991).

${ }^{8}$ D. E. Williams, J. Chem. Phys. 47, 4680 (1967).

${ }^{9}$ A. I. Kitaigorodskii, K. V. Mirskaya, and V. V. Nauchitel, Kristallografiya 9, 900 (1969).

${ }^{10}$ B. Alefeld and A. Kollmar, Phys. Lett. 57A, 289 (1976).

${ }^{11}$ A. Kollmar and B. Alefeld, Proceedings of the Conference on Neutron Scattering, Gatlinburg, MD, 1976, pp. 330-335.

${ }^{12}$ K. J. Tilli and B. Alefeld, Mol. Phys. 36, 387 (1978).

${ }^{13}$ R. M. Ibberson and M. Prager, Acta Crystallogr., Sect. B: Struct. Sci. 51, 71 (1995).

${ }^{14}$ D. Williams, Acta Crystallogr., Sect. A: Cryst. Phys., Diffr., Theor. Gen. Crystallogr. 30, 71 (1974).

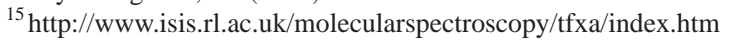

${ }^{16}$ S. F. Parker and D. J. Champion, Internet J. Vib. Spec., 3, (1999) [http:// www.ijvs.com/volume3/edition3/section1.htm\#Ed]

${ }^{17}$ M. J. Frisch, G. W. Trucks, H. B. Schlegel et al., GAUSSIAN 98, Revision A.7, Gaussian, Inc., Pittsburgh, PA, 1998.

${ }^{18} \mathrm{~J}$. Tomkinson, in Neutron Scattering From Hydrogen in Materials, edited by A. Furrer (World Scientific, Singapore, 1994), p. 168.

${ }^{19}$ V. F. Sears, Neutron News 3, 26 (1992).

${ }^{20}$ D. J. Champion, J. Tomkinson, and G. J. Kearley, Appl. Phys. (to be published)

${ }^{21}$ V. Mohacek Groev and K. Furic, J. Mol. Struct. 482-483, 653 (1999).

${ }^{22}$ M. Dörr, H. Gerlach, J. Kalus, N. Karl, M. Monkenbusch, I. Natkaniecz, U. Schmelzer, W. Schmidt, J. J. Stezowski, P. Vorderwisch, G. Voss, and M. Warth, J. Phys.: Condens. Matter 10, 1336 (1999).

${ }^{23}$ M. Monkenbusch, Comput. Phys. Commun. 67, 343 (1991). 